\title{
Why Numbers Are Embodied Concepts
}

\author{
Martin H. Fischer* \\ Cognitive Science, University of Potsdam, Potsdam, Germany
}

Keywords: arithmetic, numerical cognition, number concepts, embodied cognition, philosophy of science

Number concepts are often thought to be abstractions, for example because the numerosity of sets (e.g., their "three-ness") is a feature apparently dissociated from the sensory experiences with specific set members, such as their size, shape, or color. In other words, quantity-specific experiences seem to vary arbitrarily when we enumerate three apples, three cars, three people, or three fingers. Hence, Frege (1884) and other logically-minded philosophers considered positive integers as ideal cognitive constructions for enumerative mental operations, removed from contextual constraints, yet preserving precision and generalizing across situations (e.g., arithmetic operations).

Yet, upon closer consideration several sensory and also motor features systematically co-occur with each enumeration we perform; this co-occurrence establishes experiential patterns through which number concepts become embodied as part of their acquisition history (cf. Fischer and Brugger, 2011). I describe here several such systematic co-occurrences and cite supporting evidence. This psycho-logical view of number is not in conflict with but extends purely logical considerations of number concepts as foundations of formal arithmetic, as proposed by Frege (1884).

To-be enumerated objects are usually all simultaneously available to us and thus, by physical

OPEN ACCESS

Edited by:

Krzysztof Cipora,

Universität Tübingen, Germany

Reviewed by:

Mario Bonato,

Università degli Studi di Padova, Italy

*Correspondence:

Martin H. Fischer

martinf@uni-potsdam.de

Specialty section:

This article was submitted to

Developmental Psychology,

a section of the journal

Frontiers in Psychology

Received: 19 October 2017

Accepted: 22 December 2017

Published: 15 January 2018

Citation:

Fischer MH (2018) Why Numbers Are

Embodied Concepts.

Front. Psychol. 8:2347.

doi: 10.3389/fpsyg.2017.02347 necessity, distributed across space because two objects cannot occupy the same place at the same time. Therefore, more objects take up more space and enumerating them invites spatially distributed and temporally extended behaviors; these are sensory cues to number. The systematic directionality of counting behaviors furthermore establishes spatial-numerical associations which, in turn, can be detected with chronometric methods and through behavioral biases (see Fischer and Shaki, 2014; Winter et al., 2015, for reviews).

We know that set members should be aligned or grouped in space to reduce spatial memory load when counting them. We apply verbal sensory-motor routines to establish one-to-one correspondences between objects and number names until each object (or group) was referenced once and the last number name establishes set size or cardinality (e.g., Gelman and Gallistel, 1978). Without such direct referencing of objects through pointing, our eyes and fingers are the universal means of associating body postures (i.e., spatial, visual, kinesthetic, and proprioceptive signals) with number names (Fischer, 2003a; Di Luca and Pesenti, 2011). As a consequence, eye position predicts numerical thoughts (Loetscher et al., 2010), tactile finger stimulation primes number processing and perceiving numbers in turn modulates visual-spatial (Fischer et al., 2003) as well as tactile sensitivity (Tschentscher et al., 2012; Sixtus et al., 2017; Sixtus et al., in revision). Even when overt finger movements are avoided, we spontaneously generate repetitive upper-body movements to enrich our counting with sensory-motor feedback (Carlson et al., 2007).

Habitually, people raised in Western cultures point at horizontally distributed objects left-toright and thereby associate increasingly larger number names with increasingly more right-sided actions (Opfer and Furlong, 2011; Shaki et al., 2012). The origin of this cultural bias can be traced to observational learning at pre-school age (Göbel et al., 2018) but might have evolutionary origins (Rugani et al., 2015). In other words, the ubiquitous spatial-numerical association of response codes (SNARC) effect results from preferred sensory-motor habits. 
Two other signature effects of numerical cognition may also be embodied in origin and not only epiphenomenally so. First, when deciding which of two sets is physically larger performance is governed by Weber's law where the just-noticeable difference between them increases with set size. Moreover, we heuristically expect the larger set to also be the more numerous. This pattern is preserved when we distinguish symbolic quantities (i.e., discriminating two digits' meanings). This so-called numerical distance effect (Moyer and Landauer, 1967) suggests that we obligatorily recur to the concrete sensory and motor experiences present when these concepts were acquired.

And secondly, when gathering objects, we extend our sensorymotor experiences from the audio-visual and motor to the haptic domain. As a result, wider grasp apertures prime larger numbers (Andres et al., 2004) and number magnitude in turn biases ongoing visuo-motor control (Fischer, 2003b). Holding object sets also lets us experience positive correlations of numerosity and weight. Thus, systematic multi-modal sensorymotor experiences accompany the use of natural number concepts and pose scaled processing challenges for the cognitive system. This is the embodied foundation of the numerical size effect, i.e., the systematic increase in processing costs associated with larger numerosities, capturing most everyday experiences, such as managing to juggle 3 but not 4 balls (Fischer, 2017).

To add things up, three cardinal signatures of numerical cognition, the SNARC effect, the distance effect, and the size effect, might be grounded in sensory-motor experiences and in this sense embodied (for a terminological distinction between "grounded" and "embodied" numerical processing, see Fischer, 2012). It is therefore not surprising that we find crossdomain priming in a wide range of tasks whenever people think quantitatively, be they temporal, spatial, or conceptual (Casasanto and Boroditsky, 2008; Scheepers and Sturt, 2014; Walsh, 2015). These associations extend beyond the positive

\section{REFERENCES}

Andres, M., Davare, M., Pesenti, M., Olivier, E., and Seron, X. (2004). Number magnitude and grip aperture interaction. Neuroreport 15, 2773-2777.

Carlson, R. A., Avraamides, M. N., Cary, M., and Strasberg, S. (2007). What do the hands externalize in simple arithmetic. J. Exp. Psychol. 33, 747-756. doi: 10.1037/0278-7393.33.4.747

Casasanto, D., and Boroditsky, L. (2008). Time in the mind: using space to think about time. Cognition 106, 579-593. doi: 10.1016/j.cognition.2007.03.004

Di Luca, S., and Pesenti, M. (2011). Finger numeral representations: more than just another symbolic code. Front. Psychol. 2:272. doi: 10.3389/fpsyg.2011.00272

Fischer, M. H. (2003a). Spatial representations in number processing - evidence from a pointing task. Vis. Cogn. 10, 493-508. doi: 10.1080/13506280244000186

Fischer, M. H. (2003b). Cognitive representation of negative numbers. Psychol. Sci. 14, 278-282. doi: 10.1111/1467-9280.03435

Fischer, M. H. (2012). A hierarchical view of grounded, embodied and situated numerical cognition. Cogn. Process. 13, S161-S164. doi: 10.1007/s10339-01 2-0477-5

Fischer, M. H. (2017). "Why numbers are(n't) abstract concepts," in Presentation at the 20th Meeting of the European Society for Cognitive Psychology (Potsdam).

Fischer, M. H., and Brugger, P. (2011). When digits help digits: spatial-numerical associations point to finger counting as prime example of embodied cognition. Front. Psychol. 2:260. doi: 10.3389/fpsyg.2011.00260 integers or their manipulation in mental arithmetic (e.g., Werner and Raab, 2014) and even shape how we think about negative numbers that cannot be experienced as sensory quantities. An initial report (Fischer, 2003b) associated negative numbers with left-sided space and also showed a size effect (while controlling the distance effect). The finding generated some controversy (reviewed in Mende et al., 2017) but was confirmed when the assessment removed potential biases from spatially distributed stimulus presentation or response recording (Fischer and Shaki, 2017). Our habitual experience with spatially organized magnitudes thus replaces the lack of sensory experience with negative numbers to generate predictable sensory-motor associations.

In conclusion, number concepts, although often used in a context-free and seemingly abstract manner (Frege, 1884), always carry sensory-motor connotations. This correlative experience is used for prediction not abstraction-in other words, we apply concrete experiences gathered within a knowledge domain (the source) to generate predictions that enrich seemingly "abstract" conceptual knowledge (the target domain). Thus, it is only through the embodied lens that we can appreciate the full nature of number knowledge and devise appropriate methods for effective training and rehabilitation of numerical cognition.

\section{AUTHOR CONTRIBUTIONS}

MF: conceived of and wrote this comment and is solely responsible for the content.

\section{FUNDING}

This research was funded by the German research foundation (DFG) FI 1915/5-1 (A motor priming approach to problem solving).

Fischer, M. H., Castel, A. D., Dodd, M. D., and Pratt, J. (2003). Perceiving numbers causes spatial shifts of attention. Nat. Neurosci. 6, 555-556. doi: 10.1038/nn1066

Fischer, M. H., and Shaki, S. (2014). Spatial associations in numerical cognition: from single digits to arithmetic. Q. J. Exp. Psychol. 67, 1461-1483. doi: $10.1080 / 17470218.2014 .927515$

Fischer, M. H., and Shaki, S. (2017). Implicit spatial-numerical associations: negative numbers and the role of counting direction. J. Exp. Psychol. 43, 639-643. doi: 10.1037/xhp0000369

Frege, G. (1884). Die Grundlagen der Arithmetik (Foundations of Arithmetic). Breslau: Wilhelm Koebner.

Gelman, R., and Gallistel, C. R. (1978). The Child's Understanding of Number. Cambridge, MA: Harvard University Press.

Göbel, S. M., McCrink, K., Fischer, M. H., and Shaki, S. (2018). Observation of directional storybook reading influences young children's counting direction. J. Exp. Child Psychol. 166, 49-66. doi: 10.1016/j.jecp.2017.08.001

Loetscher, T., Bockisch, C. J., Nicholls, M. E. R., and Brugger, P. (2010). Eye position predicts what number you have in mind. Curr. Biol. 20, R264-R265. doi: 10.1016/j.cub.2010.01.015

Mende, M. A., Shaki, S., and Fischer, M. H. (2017). On the development and representation of negative number concepts (this special research topic).

Moyer, R. S., and Landauer, T. K. (1967). Time required for judgements of numerical inequality. Nature 215, 1519-1520. doi: 10.1038/2151519a0

Opfer, J. E., and Furlong, E. E. (2011). How numbers bias preschoolers' spatial search. J. Cross Cul. Psychol. 42, 682-695. doi: 10.1177/0022022111406098 
Rugani, R., Vallortigara, G., Priftis, K., and Regolin, L. (2015). Number-space mapping in the newborn chick resembles humans' number line. Science 347, 534-536. doi: 10.1126/science.aaa1379

Scheepers, C., and Sturt, P. (2014). Bi-directional syntactic priming across cognitive domains: from arithmetic to language and back. Q. J. Exp. Psychol. 67, 1643-1653. doi: 10.1080/17470218.2013.873815

Shaki, S., Fischer, M. H., and Göbel, S. M. (2012). Direction counts: a comparative study of spatially directional counting biases in cultures with different reading directions. J. Exp. Child Psychol. 112, 275-281. doi: 10.1016/j.jecp.2011.12.005

Sixtus, E., Fischer, M. H., and Lindemann, O. (2017). Finger posing primes number comprehension. Cogn. Process. 18, 237-248. doi: 10.1007/s10339-017-0804-y

Tschentscher, N., Hauk, O., Fischer, M. H., and Pulvermüller, F. (2012). You can count on the motor cortex: fMRI reveals embodied number processing. NeuroImage 59, 3139-3148. doi: 10.1016/j.neuroimage.2011.11.037

Walsh, V. (2015). "A theory of magnitude: the parts that sum to number," in The Oxford Handbook of Numerical Cognition, eds R. Cohen Kadosh and A. Dowker (Oxford: University Press), 552-565.
Werner, K., and Raab, M. (2014). Moving your eyes to solution: effects of movements on the perception of a problem-solving task. Q. J. Exp. Psychol. 67, 1571-1578. doi: 10.1080/17470218.2014.889723

Winter, B., Matlock, T., Shaki, S., and Fischer, M. H. (2015). Mental number space in three dimensions. Neurosci. Biobehav. Rev. 57, 209-219. doi: 10.1016/j.neubiorev.2015.09.005

Conflict of Interest Statement: The author declares that the research was conducted in the absence of any commercial or financial relationships that could be construed as a potential conflict of interest.

Copyright (C) 2018 Fischer. This is an open-access article distributed under the terms of the Creative Commons Attribution License (CC BY). The use, distribution or reproduction in other forums is permitted, provided the original author(s) or licensor are credited and that the original publication in this journal is cited, in accordance with accepted academic practice. No use, distribution or reproduction is permitted which does not comply with these terms. 